\title{
Tunable Lifetimes of Intramolecular Charge-Separated States in Molecular Donor-Acceptor Dyads
}

\author{
Maryam Akbarimoosavi, ${ }^{\dagger}$ Egmont Rohwer, ${ }^{\dagger}$ Ariana Rondi, ${ }^{\dagger}$ Jihane Hankache, ${ }^{\ddagger}, \|$ Yan Geng, ${ }^{\S, \perp}$ \\ Silvio Decurtins, ${ }^{\S}$ Andreas Hauser, ${ }^{\ddagger}$ Shi-Xia Liu, ${ }^{\S}$ Thomas Feurer, ${ }^{\dagger}$ and Andrea Cannizzo*, ${ }^{\dagger}$ \\ ${ }^{\dagger}$ Institute of Applied Physics, University of Bern, Sidlerstrasse 5, CH-3012 Bern, Switzerland \\ ${ }^{\ddagger}$ Department of Physical Chemistry, University of Geneva, 30 Quai Ernest Ansermet, CH-1211 Geneva, Switzerland \\ ${ }^{\S}$ Department of Chemistry and Biochemistry, University of Bern, Freiestrasse 3, CH-3012 Bern, Switzerland
}

\author{
Supporting Information
}

\begin{abstract}
We report ultrafast transient UV-vis absorption and electrochemical spectroscopies on the photoinduced charge separation dynamics in a recently synthesized family of metal-free donor-acceptor systems, where two redox-active molecules are fused into a compact and planar structure upon annulation of a tetrathiafulvalene and a benzothiadiazole as electron donor and acceptor, respectively. We found extraordinary tunability of the lifetime of the photoinduced charge separation by more than 2 orders of magnitude (from 6 to $900 \mathrm{ps}$ ) upon small changes of the peripheral residues on the acceptor and the polarity of the environment. Contrary to expectations, the lifetime of the charge separation state decreases in more polar environments and with more

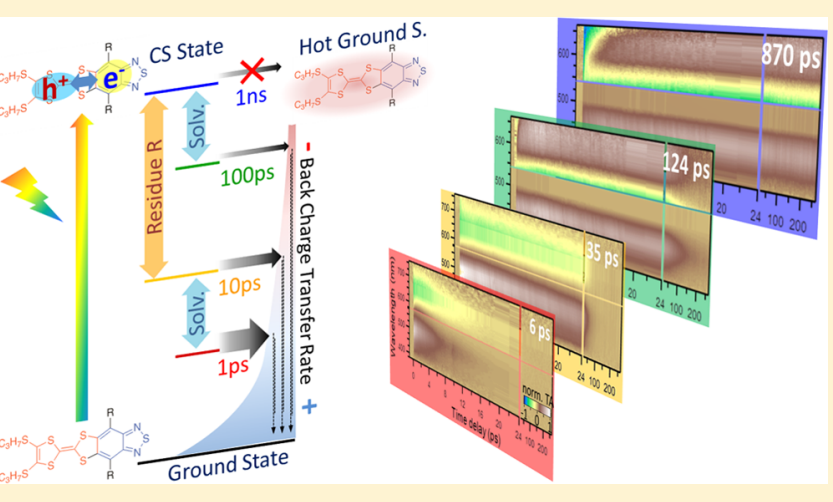
electronegative acceptors. This study proves that such fused donor-acceptor systems give rise to a new class of electronically versatile materials whose physicochemical properties can be tuned via a targeted substitution or a suitable choice of local electrostatics.
\end{abstract}

\section{INTRODUCTION}

A photosensitizer is a molecular group that by absorbing UVvis radiation triggers a physicochemical reaction in adjacent moieties or neighboring molecules by energy, electron, or charge transfer from its excited state. Photosensitization for photophysical and photochemical applications where charge or electron transfer (ET) is required, as in photovoltaics ${ }^{1-4}$ or artificial photocatalysis, ${ }^{5-13}$ is dominated by metal complexes. Coordination compounds are optically and energetically tunable, often photostable, and comparatively easy to synthesize also in big quantities. However, two points make it worthwhile to look for alternatives: (1) the low abundance on Earth of the most used metals ( Ru, Re, Pt, Ir) could set a limit to a massive usage of technologies based on them; (2) from a more fundamental point of view, the main drawback of such compounds is the presence of very efficient intersystem crossing (ISC) processes, which make the photoexcitable singlet state convert very quickly (often within tens of fs) ${ }^{14-16}$ to lower energy triplet or even quintet states. This degradation of the electronic energy into vibrational energy ultimately results in a decrease of the available driving force for the physicochemical reaction. For instance, the archetypal dye sensitizer $\left[\mathrm{Ru}(\mathrm{bpy})_{3}\right]^{2+}$ that absorbs at $450 \mathrm{~nm}(2.75 \mathrm{eV})$ relaxes in less than 40 fs to the lowest energy triplet metal-toligand charge-transfer $\left({ }^{3} \mathrm{MLCT}\right)$ state by losing $0.8 \mathrm{eV}$ of available energy because of intramolecular relaxation. Without the fast ISC process, the overall losses would be only $0.5 \mathrm{eV}$.

An alternative route consists in metal-free donor-acceptor (D-A) dyads, which typically display no ISC, small or negligible permanent electric dipole moments in the ground state (GS), and big dipole moments of up to above $10 \mathrm{D}$ in the excited state. Due to their lower cost and toxicity, higher extinction coefficients, as well as comparatively easy structural modification and tunable optical properties, they represent attractive alternatives ${ }^{17-19}$ to polypyridyl ruthenium sensitizers $^{1,2}$ and zinc-porphyrin complexes. ${ }^{3,4}$ In particular, the last decade has seen an exciting and rapid progress in the field of organic dye-sensitized solar cells (DSSCs) and in 2015, the highest power conversion efficiency (PCE) of $14 \%$ to date was reached by the cosensitization of two metal-free organic dyes. ${ }^{20}$ Although a variety of metal-free sensitizers with defined D-A architectures have been investigated to improve device performance, it remains challenging to elucidate correlations between their intrinsic properties and photovoltaic performance. $^{21,22}$ No doubt, understanding energetics and charge carrier/exciton dynamics of $\mathrm{D}-\mathrm{A}$ systems is of paramount

Received: November 14, 2018

Revised: February 6, 2019

Published: March 8, 2019 
importance to gain insight into the effective separation of the excitons in DSSCs for high PCEs. ${ }^{23}$

Tetrathiafulvalene (TTF) ${ }^{24-27}$ as an electron donor and benzothiadiazole (BTD) ${ }^{18,28,29}$ as an electron acceptor are frequently incorporated into $\mathrm{D}-\mathrm{A}$ systems for various applications in (opto)electronic devices. They can be covalently fused to form single planar $\pi$-conjugated molecules, leading to an energetically low-lying intramolecular chargetransfer (ICT) transition from the highest-occupied molecular orbital (HOMO) localized on the donor fragment to the lowest-unoccupied molecular orbital (LUMO) on the acceptor part. $^{30-32}$ The resultant HOMO-LUMO band gap can be fine-tuned by the substitution at the 4- and 8-positions of the BTD unit (Chart 1). A large red shift of the ICT absorption

Chart 1. Chemical Structures of TTF-BTD Samples

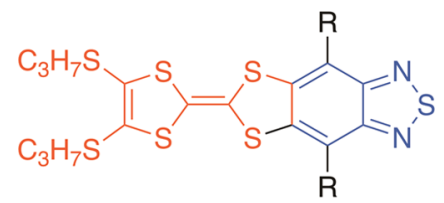

$\operatorname{TTF}-\mathrm{BTD}(\mathrm{R})_{2}$

$\mathrm{R}=\mathrm{H} ; \mathrm{Br} ; \mathrm{CN}$

band has been attributed to a significant lowering of the energy of the LUMO levels due to the strong electron-withdrawing effect of $\mathrm{Br}$ and $\mathrm{CN}$ substituents compared to that of the unsubstituted TTF-BTD $(\mathrm{H})_{2}{ }^{31}$ Consequently, TTF-BTD$(\mathrm{Br})_{2}$ behaves as a p-type semiconductor whereas TTF$\mathrm{BTD}(\mathrm{CN})_{2}$ behaves as an ambipolar semiconductor. ${ }^{30}$ This great tunability in so many physical properties, when compared with other $\pi$-conjugated systems and coordination complexes, motivates the application of such fused D-A systems in the field of molecular electronics or nonlinear optics. ${ }^{18,28,33-35}$ The study of their electronic tunability and charge-transfer characteristics is thus extremely valuable, and we recently carried out a study with Stark spectroscopy to elucidate the intimate link between the structural parameters and electronic features of the ground and excited states, such as polarizability and permanent dipoles. ${ }^{36}$ A question naturally arises: how does the tunability of the steady-state optical signals reflect on the dynamical properties of these systems? TTF and BTD states involved in the CT process are expected to be rather close in energy, possibly so close that the solvent reaction field or substitutions could significantly modulate, or even invert, the relative energies.

In a perspective view on the frontiers of molecular electronics and photocatalysis, it is evident that to achieve high photochemical performances, it is necessary to control local electrostatics and the timing with respect to other processes. Despite this, the effects of electric fields on relaxation processes are still little explored and only few articles reported spectroscopic measurements in the presence of static electric fields. ${ }^{37-43}$ These studies proved that $\mathrm{MV} / \mathrm{cm}$ fields can affect and even control relaxation processes, revealing the inadequacy to predict electric field effects on the basis of the semiclassical and single-mode Marcus theory. The seminal work of Boxer and co-workers ${ }^{40,41,44}$ on bacterial photosynthetic reaction centers triggered the development of more realistic models to explain electron transfer (ET) in photosynthetic reaction centers, which propose an electric field dependence of the rate constants of the internal conversion, which competes with the charge separation (CS) process. ${ }^{45,46}$ Tuning the timing of the local electrostatic response and of the CS state to design efficient and scalable artificial photosystems ${ }^{47,48}$ is a terrific task and requires novel photosensitizers able to generate $\mathrm{MV} / \mathrm{cm}$ local field and CS states with a welldefined timing and duration. TTF-BTD dyads have the potential to be such a system provided the lifetime of CS states of the TTF-BTD dyads show the expected tunability upon substitution and a suitable choice of the environment. Indeed, in this context, it should be noted that a dipole moment of 10 $\mathrm{D}$ creates a field of $560 \mathrm{kV} / \mathrm{cm}$ at a distance of $1 \mathrm{~nm}$, i.e., a field of $\sim 0.1-1 \mathrm{MV} / \mathrm{cm}$ on the nearby moieties.

Inspired by these questions and arguments, the electron transfer dynamics in three TTF-BTD systems have been investigated by transient absorption (TA) spectroscopy with special emphasis on the solvent and substituent effects on the charge-transfer and relaxation processes.

\section{MATERIALS AND METHODS}

Synthesis and Sample Handling. The compounds TTF$\mathrm{BTD}(\mathrm{H})_{2}{ }^{31}$ TTF-BTD $(\mathrm{Br})_{2}{ }^{32}$ and TTF-BTD $(\mathrm{CN})_{2}{ }^{30}$ were prepared according to literature procedures and dissolved in dimethylformamide (DMF), acetone, and toluene for the TA measurements and in $\mathrm{CH}_{2} \mathrm{Cl}_{2}$ for spectro-electrochemical (EC) measurements. In the following discussion, samples $\operatorname{TTF}-\mathrm{BTD}(\mathrm{R})_{2}$ will be labeled according to the residues $\mathrm{R}=$ $\mathrm{CN}, \mathrm{Br}, \mathrm{H}$.

Electrochemical Spectroscopy. Electrochemical spectra of the three samples were taken by recording UV-vis-NIR absorption spectra with an optically transparent thin layer cell $(d=0.7 \mathrm{~mm})$ from Specac, equipped with Pt working and auxiliary electrodes and a silver wire pseudoreference electrode, which was placed inside a spectrophotometer (Cary 5000), and an electrochemical potential was applied by using a potentiostat (BAS CV-50W). All solutions were freshly prepared in $\mathrm{CH}_{2} \mathrm{Cl}_{2}$ (typically $(1-2) \times 10^{-4} \mathrm{M}$ ) in the presence of $0.1 \mathrm{M} \mathrm{TBAPF}_{6}$ serving as the supporting electrolyte. All potentials are given versus the silver wire pseudoreference. The oxidation of TTF and formation of the radical cation $\mathrm{TTF}^{+}$was monitored by recording absorption spectra right after applying a positive potential of $400 \mathrm{mV}$ for a specific time. The reduction of the BTD unit was induced by applying first a negative potential of $-1250 \mathrm{mV}$ followed by $-1500 \mathrm{mV}$. Absorption spectral changes were recorded right after the application of the potential.

Transient Absorption Measurements. Samples in solution were excited close to the maximum of the respective lowest optical absorption (OA) band, namely, at 640, 520, and $490 \mathrm{~nm}$ for samples $\mathrm{CN}, \mathrm{Br}$, and $\mathrm{H}$, respectively (Figure 1 ). DMF and acetone were chosen because both are polar with comparable permanent electric dipoles (Table 1 and ref 49) but the latter has a faster solvation response ${ }^{49}$ than that of the former. Toluene can be considered as a nonpolar solvent. The solute concentrations used correspond to ODs of $\sim 0.4$ at 640 $\mathrm{nm}$ for $\mathrm{CN}, \sim 0.08$ at $520 \mathrm{~nm}$ for $\mathrm{Br}$, and $\sim 0.28$ at $490 \mathrm{~nm}$ for $\mathrm{H}$. Energy per pulse at the sample position was $50 \mathrm{~nJ}$ at $500 \mathrm{~Hz}$ and was focused into a ca. $60 \mu \mathrm{m}$ diameter spot size $\left(1 / e^{2}\right)$. To avoid photodegradation, the solutions were flowed by a gear pump through a closed flow circuit with a $200 \mu \mathrm{m}$ internal path quartz flow cell. The photostability of the samples was checked by comparing UV-vis steady-state OA measurements 


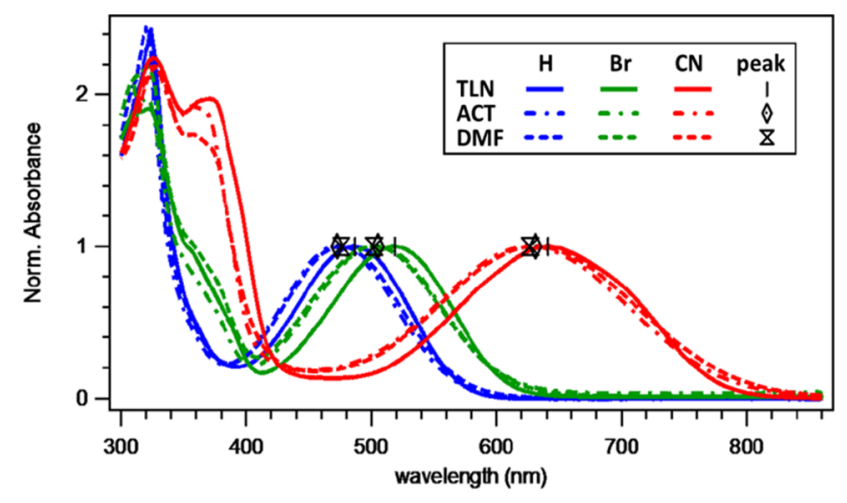

Figure 1. Steady-state OA spectra of the investigated samples in toluene, acetone, and DMF. Spectra are normalized to the peak value of the lowest band, whose position is marked for each spectrum. Samples were excited at the OA peak wavelengths $(660,525$, and 490 $\mathrm{nm}$ for $\mathrm{CN}, \mathrm{Br}$, and $\mathrm{H}$, respectively, $\left.\varepsilon_{\max }=1.5 \times 10^{4} \mathrm{M}^{-1} \mathrm{~cm}^{-1}\right)$.

Table 1. Polarizability Properties of the Solvents Relevant to This Study ${ }^{a}$

\begin{tabular}{ccccccc} 
solvent & $\mu(\mathrm{D})$ & $\varepsilon$ & $n$ & $f(\varepsilon)$ & $f(n)$ & $\Delta f$ \\
toluene & 0.3 & 2.38 & 1.50 & 0.240 & 0.226 & 0.014 \\
acetone & 2.5 & 20.7 & 1.36 & 0.465 & 0.180 & 0.285 \\
DMF & 3.7 & 36.7 & 1.43 & 0.480 & 0.205 & 0.275 \\
$a_{f(\varepsilon)=(\varepsilon-1) /(2 \varepsilon+1) ; f(n)=\left(n^{2}-1\right) /\left(2 n^{2}+1\right) ; \Delta f=f(\varepsilon)-f(n) .}$ \\
\hline
\end{tabular}

(PerkinElmer Lambda 750) immediately before and after the TA measurement.

The probe pulse was a broadband continuum at $1 \mathrm{kHz}$, generated by focusing a fraction of the Ti:Sapphire laser into a $5 \mathrm{~mm}$ thick $\mathrm{CaF}_{2}$ crystal. The pump-probe delay was controlled with a computer-controlled delay stage (Nanotec Electronic with PS 35 controller stage by OWIS GmbH) in the pump beamline.

The time resolution was 120 fs full-width at half maximum (FWHM), as measured from cross-phase modulation between pump and probe in pure solvent. A referenced detection scheme was adopted: the continuum was split into two beams of equal intensity and focused onto two different spots (ca. 40 $\mu \mathrm{m}$ diameter) on the sample. One of the two spots was overlapped with the pump pulse, whereas the other was kept unperturbed for referencing. Thereafter, the sample probe and reference are collimated by an achromatic doublet lens, dispersed by a grating, and then focused into two fast CMOS cameras capable of measuring individual shots (Glaz PulseSync, Synertronic Designs), allowing shot-by-shot correction of spectral and amplitude fluctuations of the continuum and of the pump. The relative polarization between pump and probe is adjusted to be parallel. A power dependence measurement was regularly carried out to ensure that experiments are conducted in the linear absorption regime.

More information on the setup, on the calculation of the signal, and on the correction of spectral and amplitude fluctuations is reported in the Supporting Information (SI).

Data Analysis. After correction for probe group velocity dispersion, data from -150 to 150 fs around time zero were neglected to avoid artifacts caused by pump-probe crossphase modulation from the solvent. A singular value decomposition and global fit (SVD-GF) analysis ${ }^{14}$ on the transient absorption data $\operatorname{TA}(\lambda, t)$ was performed to separate the noise, $\Xi(\lambda, t)$, from the spectral evolution of the system, $\widehat{\mathrm{TA}}(\lambda, t)$

$$
\mathrm{TA}(\lambda, t)=\widehat{\mathrm{TA}}(\lambda, t)+\Xi(\lambda, t)
$$

and to decompose the latter as an expansion of exponential decays with characteristic lifetimes $\left(\tau_{k}\right)$ and decay associated spectra (DAS)

$$
\widehat{\mathrm{TA}}(\lambda, t)=\left[\sum_{k=1}^{p} \mathrm{DAS}_{k}(\lambda) \cdot \mathrm{e}^{-\left(t / \tau_{k}\right)} \cdot u(t)\right] \otimes \mathrm{e}^{-\left(t-t_{0} / 0.6 \Delta_{\mathrm{IRF}}\right)^{2}}
$$

where $u(t)$ is the Heaviside step function and the expansion is convoluted with a Gaussian instrumental response function (IRF) with an FWHM of $\Delta_{\text {IRF }}$ and centered at $t_{0}$. See the SI for more details.

\section{RESULTS AND DISCUSSION}

Steady-State Absorption. Figure 1 shows OA spectra of the investigated samples in toluene, acetone, and DMF, ordered according to increasing permanent electric dipole and dielectric constant (Table 1 and ref 49). All samples show a clear band in the visible range that undergoes a blue shift moving through the series $\mathrm{R}=\mathrm{CN}, \mathrm{Br}$, and $\mathrm{H}$. With an extinction coefficient of around $10^{-4} \mathrm{M}^{-1} \mathrm{~cm}^{-1}$ and an oscillator strength of around 0.1 , this band has been assigned to an optically allowed transition to the lowest singlet excited state showing an intramolecular charge-transfer character from the TTF to the BTD moiety. ${ }^{30-32,36}$

Notably, for the more polar solvents, the ICT band is blueshifted compared with toluene, for all three compounds. At a first glance, this would suggest that the excited state is less polar than the ground state but a proper reading of this result needs to consider explicitly all contributions to the solventsolute interaction. The effect of the solvent response on the transition frequency can be written as ${ }^{50}$ (see the SI)

$$
h \nu=h \nu_{\mathrm{V}}-\left(\mu_{\mathrm{E}}-\mu_{\mathrm{G}}\right) \frac{2}{a^{3}}\left[\mu_{\mathrm{G}} \Delta f+\left(\mu_{\mathrm{E}}+\mu_{\mathrm{G}}\right) f(n)\right]
$$

where $h \nu$ is the ground (G)-to-excited (E)-state transition frequency upon photon absorption; $h \nu_{\mathrm{V}}$ is the transition frequency in vacuum; $\mu_{\mathrm{G} / \mathrm{E}}$ is the permanent electric dipole of the $\mathrm{G} / \mathrm{E}$ state, respectively; and $a$ is the radius of the Lorentz cavity in which the solute resides and its value can be set equal to the molecular size. $f(n)=\left(n^{2}-1\right) /\left(2 n^{2}+1\right)$ and $f(\varepsilon)=(\varepsilon$ $-1) /(2 \varepsilon+1)$ are the high- and low-frequency polarizabilities of the solvent, respectively, and $\Delta f=f(\varepsilon)-f(n)$ is the orientation polarizability, which describes the effect of permanent dipoles of the solvent; $f(n)$ and $f(\varepsilon)$ are normally positive. The polarizability properties of the used solvents are summarized in Table 1.

As expected, toluene, the apolar solvent, has a negligible orientation term, but its electronic polarizability contribution is bigger than that for the other two solvents. From eq 3 and values in Table 1 , we can easily derive that the observed dependence of the band shift on the solvent is compatible with a $\mu_{\mathrm{G}} \approx 0$ only. This implies that the main contribution to the transition frequency shift originates from the instantaneous electronic polarizability $f(n)$ and not from the orientation term $\Delta f$. This special condition makes the shift in toluene the largest. It is remarkable that a $\mu_{\mathrm{G}}>\mu_{\mathrm{E}} / 10$ is enough to reestablish the ordinary behavior. This is in excellent agreement with computational results and from Stark spectroscopy. ${ }^{36}$ 

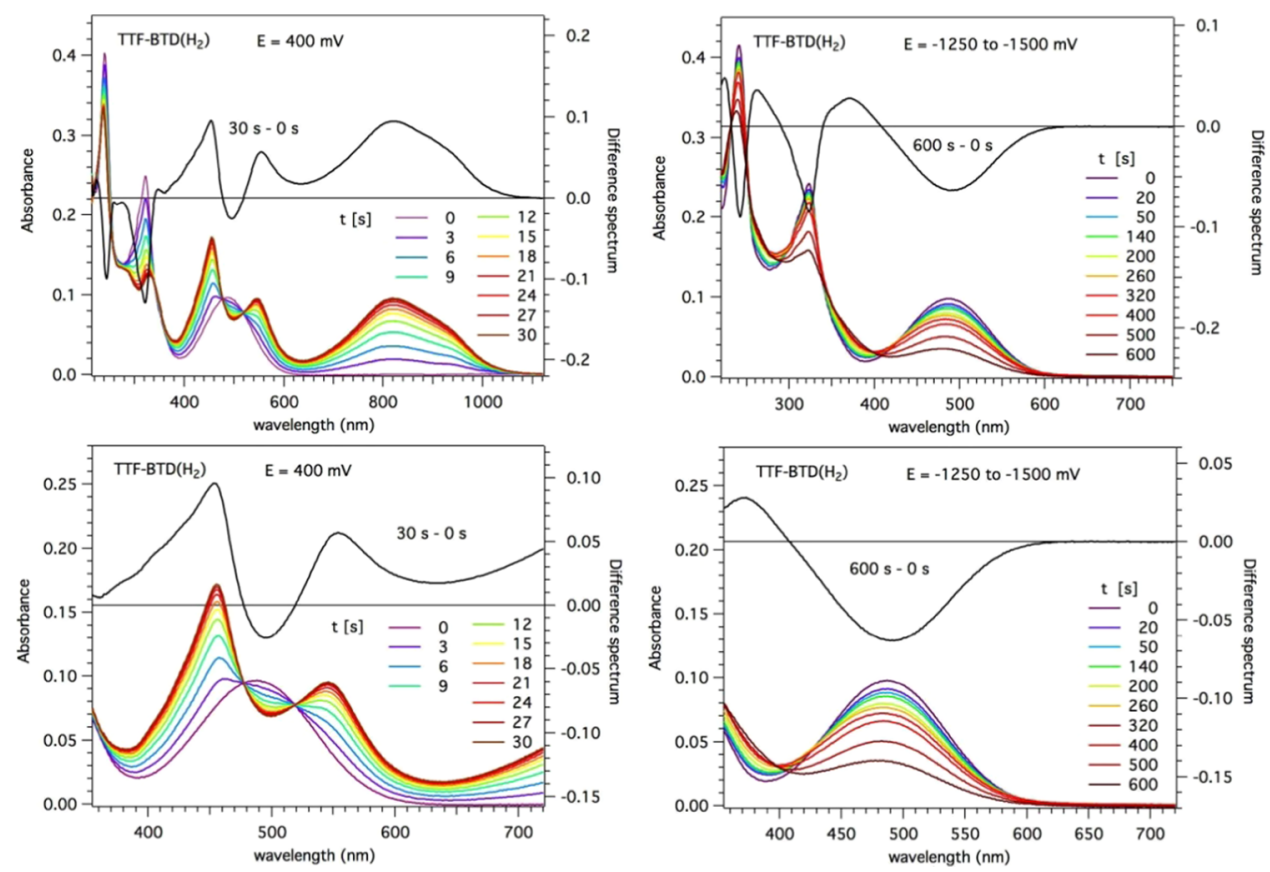

Figure 2. Electrochemical spectroscopy of TTF-BTD $(\mathrm{H})_{2}$. Left panels show absorption spectral changes corresponding to a progressive oxidation of the TTF moiety. Right panels show a progressive reduction of the BTD unit. The black curves represent the differential spectrum between the fully one-electron oxidized (reduced) molecule and the neutral one. Bottom panels show the portion of the respective top panel in the spectral range relevant to transient absorption measurements. The same plots for samples $\mathrm{Br}$ and $\mathrm{CN}$ are shown in Figures S1 and S2.

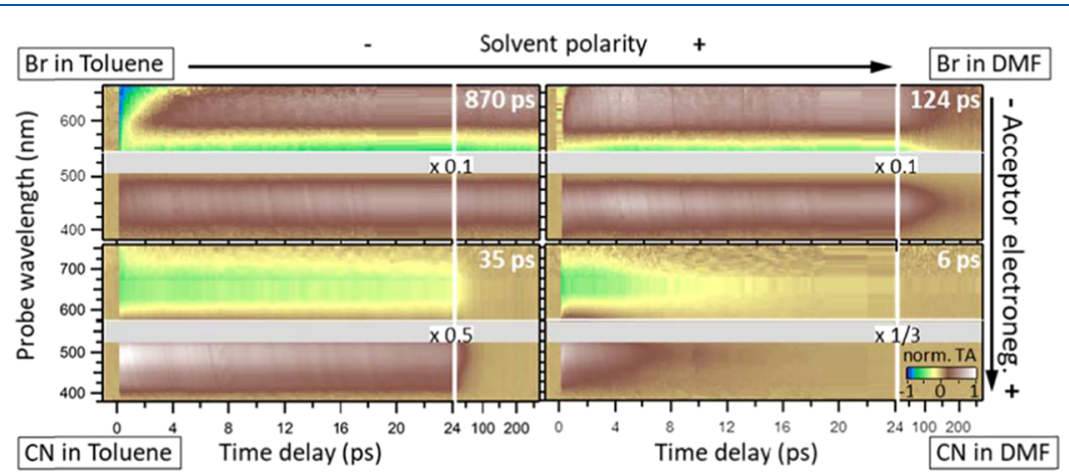

Figure 3. Time-wavelength transient absorption 2D plots of TTF-BTD $(B r)_{2}$ and TTF-BTD $(C N)_{2}$ (i.e., with a less and more electronegative acceptor, respectively) in toluene and DMF (nonpolar and polar solvents, respectively). For the sake of clarity, data are normalized to the maximum (see Figures 7 and 8 for amplitudes); at $\lambda<550 \mathrm{~nm}$, they are multiplied by a suitable value (black value on the respective shadowed area) and time axes are split in two intervals (0-24 and 24-270 ps) to appropriately show the earlier dynamics. The spectral region contaminated by pump scattering is shadowed. White numbers on each plot are excited-state (or charge separation) lifetimes according to data analysis (see Table 2 and the relative discussion). Black arrows are a guide for the reader to show the effect of solvent polarity and acceptor electronegativity on the shortening of the charge-separated state.

Spectro-electrochemistry. In a simplified picture, the effect of a photoinduced ICT transition is to oxidize the TTF and reduce the BTD groups simultaneously within the same molecule. Although transitions involving delocalized and higher ICT states could be affected in a nontrivial way, we could expect that transient excited-state absorptions (ESAs) due to transitions localized on either of the two moieties could resemble a combination of absorption bands of electrochemically oxidized TTF and reduced BTD, similar to what was found for the electronic absorption spectra of MLCT-excited states of bipyridine and phenanthroline metal complexes. ${ }^{51}$ Figure 2 shows the effect of a one-electron oxidation and reduction of the TTF and BTD groups, respectively, on the spectrum of the $\mathrm{H}$ compound. To compare these measurements with TA signals in the next section, we observe that the latter are difference spectra, which show spectral changes with respect to the GS absorption of the sample upon optical excitation. Then, a convenient way to compare the electrochemical (EC) spectra with TA spectra is to also calculate difference EC (DEC) spectra by subtracting the EC spectrum at $t=0 \mathrm{~s}$ from the one corresponding to a fully oxidized/ reduced species (gray lines in Figure 2). Figures S1 and S2 show the same plots for the $\mathrm{Br}$ and $\mathrm{CN}$ compounds, respectively.

Focusing on the spectral region relevant to the comparison with TA measurements (350-720 nm), we recognize in the $\mathrm{H}$ sample that the main effect in the radical cation $\mathrm{TTF}^{\bullet+}$ is the appearance of a DEC band at $440 \mathrm{~nm}$. In addition, a rather broad DEC band appears at $\lambda>550 \mathrm{~nm}$ and a negative DEC signal is observed at $\sim 500 \mathrm{~nm}$ due to the bleaching of the OA 
A
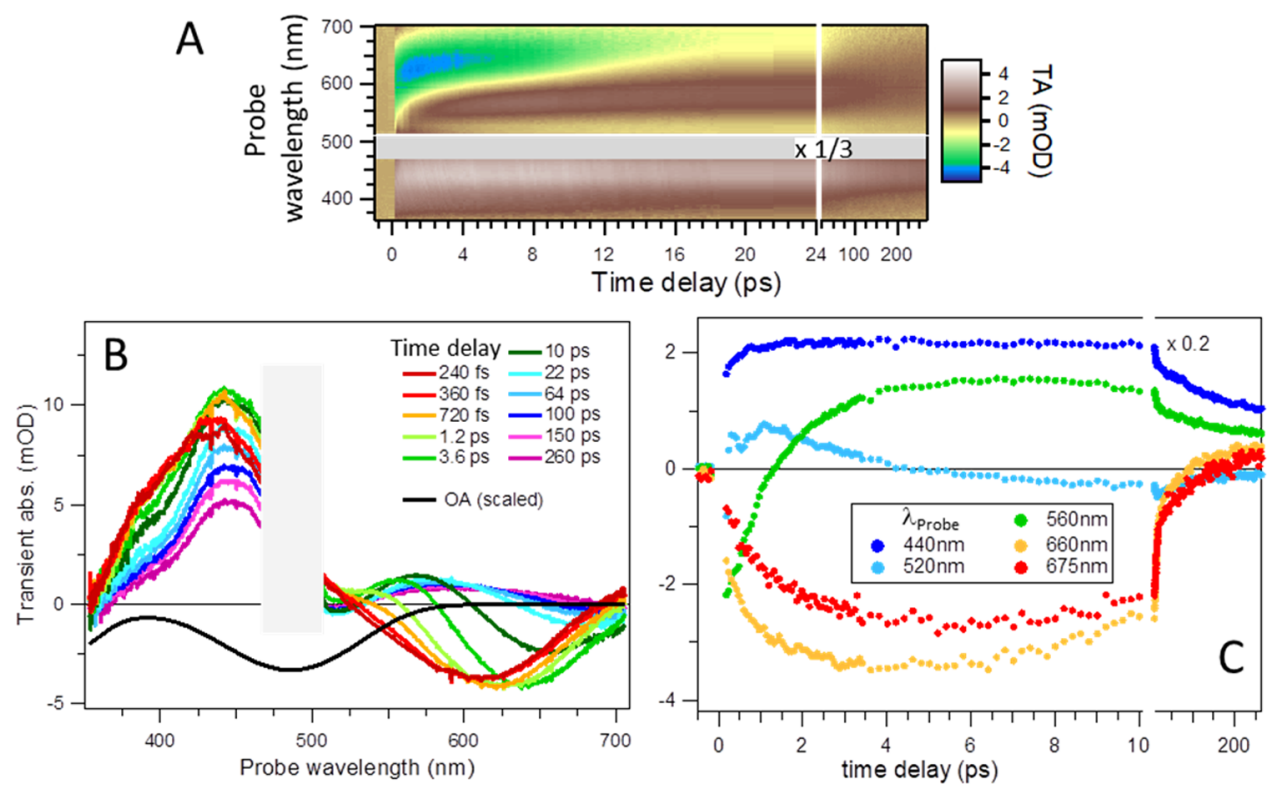

Figure 4. Transient absorption of sample $\mathrm{H}$ in toluene upon excitation at the maximum of the first electronic OA band (i.e., $490 \mathrm{~nm}$, see Figure 1). Panel (A) shows the time-wavelength 2D color plot. For the sake of clarity, data at $\lambda<500 \mathrm{~nm}$ are divided by 4 and the time axis is split into two intervals (0-24 and 24-270 ps) to appropriately show the earlier dynamics. A representative selection of spectra at different times and kinetic trace at different wavelengths is reported in panels (B) and (C), respectively. The spectral region contaminated by pump scattering at $490 \mathrm{~nm}$ is hidden in panels (A) and (B). Panel (B) shows also the steady-state OA band, inverted and scaled.

spectrum of the neutral species. The DEC spectrum of the reduced $\mathrm{BTD}^{\bullet-}$ consists mainly of a positive signal at $\lambda<400$ $\mathrm{nm}$ and a negative bleaching of the $\mathrm{OA}$ band of the neutral species. For the reduced species, no signal is observed at $\lambda>$ $500 \mathrm{~nm}$.

The Br sample shows a similar behavior with a global red shift of 20-30 nm. The CN compound displays an analogous pattern red-shifted by $100-120 \mathrm{~nm}$. This shift causes the lower energy DEC band to disappear from the detection window relevant to the TA measurements, making DEC spectra of $\mathrm{TTF}^{\bullet+}$ and $\mathrm{BTD}^{\bullet-}$ less distinguishable.

Transient Absorption. A graphical summary of the TA results is shown in Figure 3 where the four representative cases (acceptor with high and low electronegativity in polar and nonpolar solvent) are presented in the form of a wavelengthtime two-dimensional (2D) plot (a complete summary is reported in Figure S3). A complex spectral pattern and dynamical behavior are observed in all samples, which will be discussed and rationalized in the next sections, but one of the main results of this work is already clearly observable beyond any analysis or interpretation: the outstanding tunability of the excited-state (namely, of charge separation) lifetime of such a family of D-A dyads upon minimal changes in the acceptor periphery and the solvent polarity. Before discussing the origin of such a behavior, we will first discuss in detail TTF-BTD $(\mathrm{H})_{2}$ in toluene because it represents more distinctly the behavior of all other samples. Then, we will outline the effects of the solvent on the photocycle of this sample. In Conclusions of this section, we will study the effect of changing the $\mathrm{H} / \mathrm{Br} / \mathrm{CN}$ substituent on the BTD ligand.

Photocycle and Solvent Effects: TTF-BDT $(\mathrm{H})_{2}$ in Different Solvents. The wavelength-time $2 \mathrm{D}$ plot in Figure $4 \mathrm{~A}$ presents the spectral evolution of $\operatorname{TTF}-\mathrm{BTD}(\mathrm{H})_{2}$ in toluene. A selection of TA spectra at different pump-probe time delays (panel B) and kinetic traces measured at selected probe wavelengths (panel C) are also shown. A strong excited- state absorption (ESA) at $440 \mathrm{~nm}$ develops within the excitation pulse, undergoes a limited narrowing and a red shift in less than $500 \mathrm{fs}$, and afterward it slowly decays with no further spectral evolution (kinetic trace at $440 \mathrm{~nm}$ in Figure 4C). In contrast, the dynamics in the range of $\lambda>500 \mathrm{~nm}$ are more complex: the earliest spectra show a strong negative signal covering all of this range, which undergoes a red shift from 610 to $680 \mathrm{~nm}$ in less than 3 ps (Figure $4 \mathrm{~B}, \mathrm{C}$ at $\lambda>650$ $\mathrm{nm})$. The comparison with the continuous wave (cw) OA band clearly rules out that this negative signal is a ground-state bleach (GSB) but a stimulated emission (SE) signal, very likely corresponding to the inverse transition of the $\mathrm{cw}$ OA at 490 $\mathrm{nm}$. The SE signal, initially centered at ca. $600 \mathrm{~nm}$, undergoes a red shift to ca. $675 \mathrm{~nm}$, uncovering a weak negative signal at $500 \mathrm{~nm}$ and an ESA at 570-600 $\mathrm{nm}$. Whereas the former can be assigned to a GSB signal, the assignment of the latter relies on the comparison in the next section to the electrochemical spectra.

According to calculations and previous spectroscopic studies, ${ }^{36}$ the excited state has a strong CT character and the decay of the ESA at $440 \mathrm{~nm}$ and SE at $660 \mathrm{~nm}$ can be used as reporters of the back-charge-transfer (BCT) process, whereas the GSB signal at $525 \mathrm{~nm}$ tracks the recovery to the pristine electronic configuration. Kinetic traces reveal that the CT state lasts for several hundreds of $p$ s and the presence of an SE at $600-700 \mathrm{~nm}$ confirms that the BCT is, at least partially, radiative, in line with the reported luminescence for the $\mathrm{Br}$ compound. $^{32}$ The outcome of the SVD-GF analysis is summarized in Table 2 and Figure S6: the red shift of the SE and the narrowing of the $440 \mathrm{~nm}$ ESA is a triphasic process with $0.46,3.3$, and 7.28 ps time constants.

To clarify the role of the solvent and to have an insight on the nature of the excited states and of these dynamics, we carried out the same measurements in the two polar solvents $\mathrm{DMF}$ and acetone. Figure 5 shows results for the $\mathrm{H}$ sample in the more polar DMF. A similar behavior was observed in 
Table 2. Time Constants $\left(\tau_{n}\right)$ of the Relevant Dynamics Obtained by SVD-GF Analysis of the Time-Wavelength Transient Absorption Data from Figures 4,7,8, and S4 (See Also Figure S3) ${ }^{a}$

\begin{tabular}{|c|c|c|c|}
\hline & DMF (ps) & acetone (ps) & toluene (ps) \\
\hline \multicolumn{4}{|c|}{ TTF-BTD $(\mathrm{H})_{2}$} \\
\hline$\tau_{1}[\mathrm{CSS}]$ & $0.04 \pm 0.01$ & & \\
\hline$\tau_{2}[\mathrm{CSS}+\mathrm{SS}]$ & $0.30 \pm 0.01$ & $0.41 \pm 0.02$ & $0.46 \pm 0.03$ \\
\hline$\tau_{3}[\mathrm{SS}+\mathrm{VET}]$ & $1.61 \pm 0.04$ & $1.60 \pm 0.10$ & $3.30 \pm 0.12$ \\
\hline$\tau_{4}[\mathrm{SS}+\mathrm{VET}]$ & & & $7.28 \pm 0.12$ \\
\hline$\tau_{5}[\mathrm{BCT}+\mathrm{Rot}]$ & $127 \pm 10$ & $54 \pm 4$ & $220 \pm 50$ \\
\hline$\tau_{6}[\mathrm{BCT}]$ & $570 \pm 200$ & $480 \pm 30$ & $410 \pm 50$ \\
\hline \multicolumn{4}{|c|}{ TTF-BTD $(\mathrm{Br})_{2}$} \\
\hline \multicolumn{4}{|l|}{$\tau_{1}[\mathrm{CSS}]$} \\
\hline$\tau_{2}[\mathrm{CSS}+\mathrm{SS}]$ & $0.74 \pm 0.08$ & $0.22 \pm 0.06$ & \\
\hline$\tau_{3}[\mathrm{SS}+\mathrm{VET}]$ & $\sim \tau_{2}$ & $1.18 \pm 0.15$ & $1.05 \pm 0.10$ \\
\hline$\tau_{4}[\mathrm{SS}+\mathrm{VET}]$ & & & $7.9 \pm 0.7$ \\
\hline$\tau_{5}[\mathrm{BCT}+\mathrm{Rot}]$ & $124 \pm 1$ & $110 \pm 40$ & $40 \pm 9$ \\
\hline$\tau_{6}[\mathrm{BCT}]$ & $\sim \tau_{5}$ & $560 \pm 300$ & $870^{32}$ \\
\hline \multicolumn{4}{|c|}{ TTF-BTD $(\mathrm{CN})_{2}$} \\
\hline$\tau_{1}[\mathrm{CSS}]$ & $0.06 \pm 0.04$ & $0.24 \pm 0.02$ & $0.76 \pm 0.06$ \\
\hline$\tau_{2}[\mathrm{CSS}+\mathrm{SS}]$ & $0.43 \pm 0.07$ & $2.18 \pm 0.14$ & $2.5 \pm 0.4$ \\
\hline$\tau_{3}[\mathrm{SS}+\mathrm{VET}]$ & $3.7 \pm 1.8$ & & \\
\hline \multicolumn{4}{|l|}{$\tau_{4}[\mathrm{SS}+\mathrm{VET}]$} \\
\hline$\tau_{5}[\mathrm{BCT}+\mathrm{Rot}]$ & & & $35.1 \pm 0.2$ \\
\hline$\tau_{6}[\mathrm{BCT}]$ & $6.12 \pm 0.07$ & $5.72 \pm 0.04$ & $\sim \tau_{5}$ \\
\hline
\end{tabular}

${ }^{a}$ Decay associated spectra (DAS) are reported in the SI (Figures S6S8). Those time constants that are so close to being indistinguishable are marked with $\sim \tau_{n}$. For the sake of clarity, time constants are also labeled according to the respective underlying physical process assigned in the text (CSS = charge separation and stabilization SS = Stokes shift, $\mathrm{VET}=$ vibrational energy transfer to the solvent, $\mathrm{BCT}=$ back charge transfer, and Rot = rotational diffusion).

acetone (Figures S3 and S6), and only results in DMF will be shown in detail. The comparison with data in Figure 4 reveals the following: (i) ESA at $440 \mathrm{~nm}$ is almost unaffected by a polar environment; (ii) the SE undergoes a much more pronounced red shift, so large as to leave the detection window $(\lambda>700 \mathrm{~nm})$ in less than $2 \mathrm{ps}$; (iii) also, 550-700 nm ESA bands on longer times are weakly affected, and the main difference at $\lambda>650 \mathrm{~nm}$ can be ascribed to the presence of SE in toluene. Kinetic traces at the peak of the blue ESA band are plotted together in Figure 6 to compare the overall lifetime of

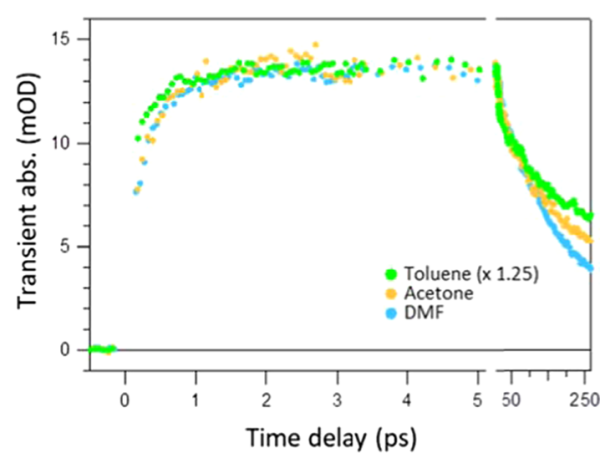

Figure 6. Kinetic traces at the maximum of the ESA band $(440 \mathrm{~nm})$ in toluene (green), acetone (orange), and DMF (blue).

the CT state in the three solvents (see also Table S1). Earlier rise dynamics in toluene are definitively faster and less pronounced than the ones in polar solvents, whereas on moving to longer dynamics, the overall lifetime of the CT state is substantially shortened in polar solvents. This is indeed confirmed by the outcome of the data analysis reported in Table 2 and Figure S6: although the behavior in the two polar solvents is virtually the same, we observe with respect to toluene a shortening of the longest components and an increase of the DAS amplitudes of the fastest dynamics $\left(\tau_{1}\right.$, when present, and $\tau_{2}$ ). This rise shows a complex multiphasic behavior, and to clarify the different contributions, we calculated the zeroth spectral moment, $M_{0}$, of the $440 \mathrm{~nm}$ ESA band as a function of time (eq S10), integrating over the
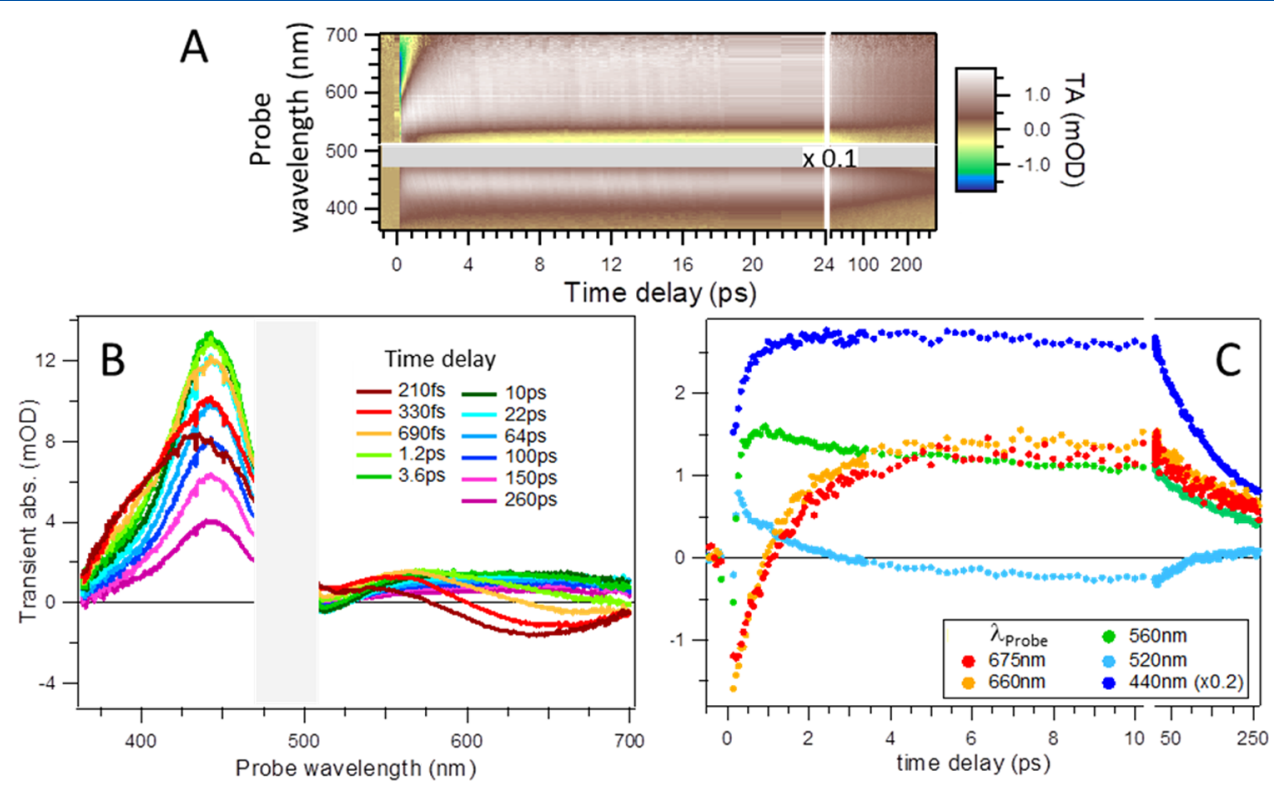

Figure 5. Transient absorption of sample $\mathrm{H}$ in DMF upon the same experimental conditions of the experiment in toluene (Figure 4). Panel (A), (B), and (C) show a time-wavelength $2 \mathrm{D}$ color plot, a representative selection of spectra at different times, and kinetic trace at different wavelengths, respectively (see the caption of Figure 4). The spectral region contaminated by pump scattering is shadowed in panels (A) and (B). 


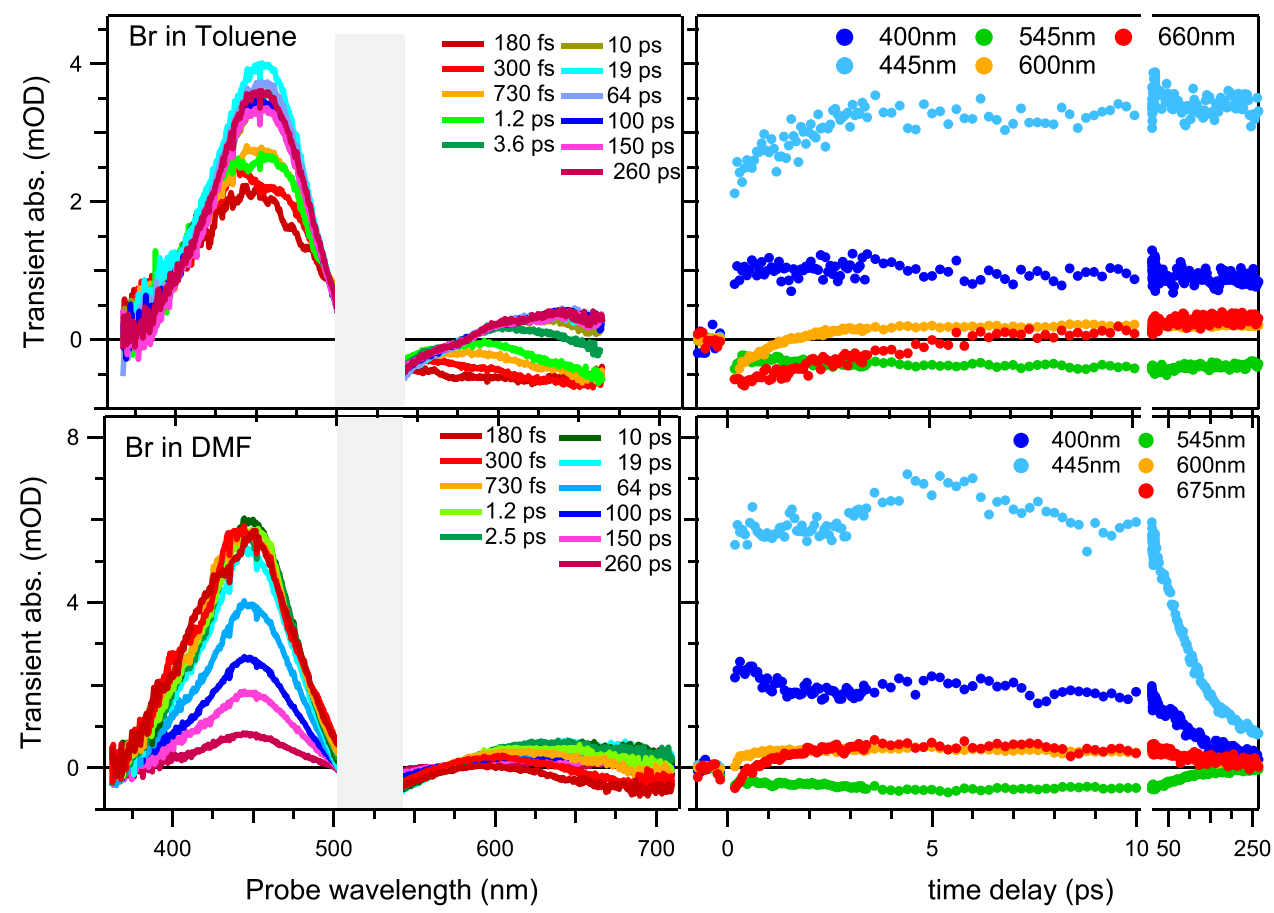

Figure 7. Transient absorption of sample $\mathrm{Br}$ in toluene and DMF upon excitation at the maximum of the first electronic OA band (i.e., $525 \mathrm{~nm}$, see Figure 1). Left and right panels show a representative selection of spectra at different times and kinetic traces at different wavelengths, respectively (see also top panels of Figure 3). The spectral region contaminated by pump scattering at $525 \mathrm{~nm}$ is hidden in left panels.

interval $510-350 \mathrm{~nm}$ to cover the entire ESA band. $M_{0}$ is supposed to be proportional to the number of excited molecules. This can be accepted even in the present case where the ESA signals within the integration interval could be slightly contaminated by GSB contributions and distortion due to the pump scattering.

We observe that picosecond dynamics (described by $\tau_{3}$ and $\tau_{4}$ ) disappear from $M_{0}$ traces, revealing that the rise is mainly due to the narrowing of the band and the subsequent increase of the peak amplitude (see Figure S10). However, a rise component is still present, which is more relevant in polar solvents. The time scale of this component agrees well with the values of $\tau_{1}$ and $\tau_{2}$. Since it is observed in the nonpolar toluene but is more evident in the polar ones, we ascribe it to an intramolecular process of formation and stabilization of the phototransferred electron on the acceptor moiety: such a process can indeed be modulated both by slow conformational changes of the molecules (always present regardless of environment) and by the solvent reaction field. ${ }^{52,53}$ Conversely, $\tau_{3}$ and $\tau_{4}$ picosecond components can be ascribed to Stokes shift and cooling, in agreement with their absence in $M_{0}$ traces and the peculiar spectral changes (narrowing of the 440 $\mathrm{nm}$ band and red shift of the 550-700 nm ESA) observed at $t$ $>10 \mathrm{ps}$ and in the corresponding DASs (Figure S6). Their shortening in polar solvents can be rationalized as not only due to a stronger coupling with the solvent (mainly observable at the $440 \mathrm{~nm}$ ESA band) but also the departure from the detection window of the SE signal.

Moving to $\tau_{5}$ and $\tau_{6}$ and the respective DASs, they describe a decay of the signal at $440 \mathrm{~nm}$ and at $550-700 \mathrm{~nm}$, with no changes in the shape of these bands. Spectral evolution corresponding to $\tau_{5}$ mainly describes a decrease of ca. $2 / 3(4 / 5$ in DMF) of the $440 \mathrm{~nm}$ band in $100 \mathrm{~s}$ ps, with no changes in spectral profiles (compare plots " $\tau_{5}+\tau_{6}$ ” and “ $\tau_{6}$ ” in Figure S6). This is typical of rotational diffusion, and thus we believe that such a process is a main cause of this component. A decrease of $2 / 3$ speaks for an ESA transition dipole moment perfectly aligned to the probe at time zero. In our case (pump and probe with parallel polarizations), this also implies that it is parallel to the one responsible for the GS absorption.

Noteworthily, the extinction ratio of the 550-700 nm ESA band in polar solvents (in toluene, this band is affected by a residual SE band) is smaller by a factor of 2 , pointing to a nonparallel orientation by $35^{\circ}$ of the transition dipole moment of this band with respect to the other two. However, the presence in toluene of intramolecular dynamics in hundreds of ps, as revealed by the $S E$ evolution at $\lambda>600 \mathrm{~nm}$, a drop of the band in DMF to $1 / 5$, which is by far more than the expected $1 / 3$, and the comparison with other samples (see the next section) strongly suggest that $\tau_{5}$ dynamics are also due to intramolecular electronics processes such as BCT. BCT is also the natural rationalization for the mechanism underlying $\tau_{6}$ : according to the previous argumentation about the nature of the two ESA bands, $\tau_{6}$ is assigned to BCT and recovery of the pristine charge distribution. This experimental behavior suggests a rather simple photocycle (Figure S11): excitation of the ground state (GS) into the lowest allowed transition produces the lowest singlet excited state $S_{1}$ identified as TTF $\rightarrow$ BTD ${ }^{1}$ ICT. The extent of the charge separation and stabilization of the phototransferred electron on the acceptor moiety increases in the course of the sub-picosecond intramolecular relaxation process and solvent dielectric reorganization $\left(\tau_{1}\right.$ and $\left.\tau_{2}\right)$. Vibrational relaxation occurs on a picosecond time scale by intramolecular conformational relaxation and vibrational energy transfer to the solvent $\left(\tau_{3}\right.$ and $\left.\tau_{4}\right)$.

The charge separation excited-state decays with an almost nanosecond lifetime $\left(\tau_{6}\right)$, which makes radiative charge recombination an important, if not the principal, BCT mechanism. This is modulated by a 100 ps rotational diffusion 


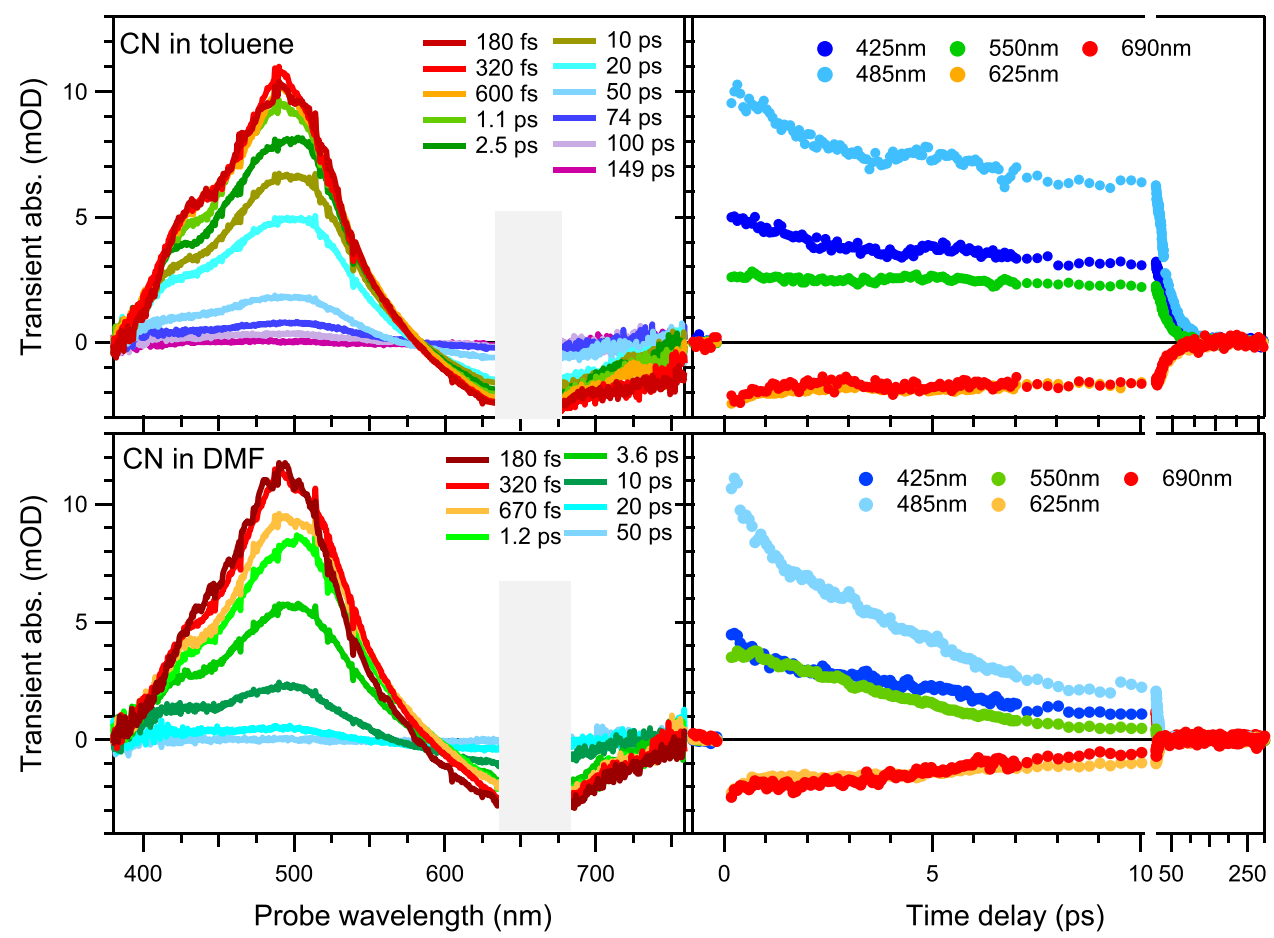

Figure 8. Transient absorption of sample $\mathrm{CN}$ in toluene and DMF upon excitation at the maximum of the first electronic OA band (i.e., $660 \mathrm{~nm}$, see Figure 1). Left and right panels show a representative selection of spectra at different times and kinetic trace at different wavelengths, respectively (see also bottom panels of Figure 3). The spectral region contaminated by pump scattering at $525 \mathrm{~nm}$ is hidden in left panels. Small fluctuations of the signal in toluene and DMF observed in the kinetics at 5 and 10 ps, respectively, are due to laser instability.

and slow electronic relaxations $\left(\tau_{5}\right)$. Concerning the effect of solvent polarity, it is 2-fold: (1) vibrational and electronic relaxations are faster and larger in polar environment; (2) there is a shortening of the CS lifetime and a stronger coupling with nonradiative BCT relaxation channels, which involve vibrationally or electronically excited levels of the system in a nonCS state.

This cycle is qualitatively valid for all investigated complexes, although with quantitative differences in the kinetics and quantum yields (Table S1).

Back-Charge-Transfer Process and Charge Separation Lifetime. To clarify the relevance of the electronegativity of the acceptor moiety on the stability of the CS state, we compare the photoinduced dynamics of $\mathrm{Br}$ and $\mathrm{CN}$ complexes with the $\mathrm{H}$ one in the three solvents. With the same argument, we will discuss in detail results in toluene and DMF, since behavior in acetone is qualitatively similar to that in DMF (see Figures S3, S4 and S7, S8).

Figures 7 and 8 show a representative selection of timeresolved spectra and single-wavelength kinetics of samples $\mathrm{Br}$ and $\mathrm{CN}$, respectively, in toluene and DMF. The outcome of respective SVD-GF analyses is reported in Table 2 and Figures S7 and S8. Similar to the previous assignments for the sample $\mathrm{H}$, sample $\mathrm{Br}$ shows at early times an SE signal due to the inverse transition responsible for the lowest $\mathrm{cw}$ OA band at $525 \mathrm{~nm}$ (Figure 1). Conversely, the negative signal of the sample $\mathrm{CN}$ is a GSB corresponding to the cw OA band at 660 $\mathrm{nm}$ (Figure 1). In analogy with the analysis of the $\mathrm{H}$ sample, we compare first measurements in toluene, since the lack of dielectric relaxation makes the identification of intramolecular mechanisms more conclusive (for the sake of argument, the experimental data and relative analyses are grouped together in Figures S5 and S9, respectively).
As expected from cw OA spectra in Figure 1, sample $\mathrm{Br}$ behaves rather comparably as the $\mathrm{H}$ one, with the main difference in the spectral position of SE, which shifts following the new peak position of the respective $\mathrm{cw}$ OA band (Table S2). ESA bands are almost unaffected by the substitution of $\mathrm{H}$ with $\mathrm{Br}$, since the apparent red shift of the $600 \mathrm{~nm}$ ESA band in $\mathrm{Br}$ is probably not real but due the GSB shift from 490 to $525 \mathrm{~nm}$, going from $\mathrm{H}$ to $\mathrm{Br}$. Noteworthily, the lifetime of the CS state in the Br sample is, within our scanned time interval, much longer than that in the $\mathrm{H}$ complex with less dynamics in the $100 \mathrm{ps}$ time scale. This implies that $\tau_{5}$ component is not purely rotational. Moving to the $\mathrm{CN}$ sample, we observe only the higher energy ESA, now at $490 \mathrm{~nm}$, and GSB at $\sim 650 \mathrm{~nm}$, both excellent markers of the BCT process. The effect of the $\mathrm{CN}$ substitution on the CS lifetime is dramatic with a shortening from $870^{32}$ to 35 ps. A closer look at the earlier dynamics in Figure 8 reveals a small narrowing on the red tail of the GSB in 2.5 ps $\left(\tau_{2}\right)$, which is compatible with a residual SE contribution exiting the detection window (see also SI.8).

Remarkably, from the analysis of the experimental data reported herein and taken from the literature, ${ }^{32}$ we can also estimate the positions of the relaxed ( $t>$ tens of ps) emission peak $\left(\lambda_{\text {Max }}^{\mathrm{Em}}\right)$ for all samples both in toluene and DMF, as listed in Table 3 (for more details, see the table caption and Section SI.8). This allows us to order the samples according to the energy gap between the equilibrated excited state and the ground one.

It is worth mentioning that all samples show the same subpicosecond rise of the overall signal already commented on in the previous section. Accordingly, this process and the picosecond Stokes shift of the SE are intramolecular processes, which involve an increase of the extent of the charge separation 
Table 3. Positions of the Emission Peak $\left(\lambda_{\mathrm{Max}}^{\mathrm{Em}}\right)$ and the Pure (i.e., Without Contributions from Rotational Diffusion) Back-Charge-Transfer (BCT) Times $\left(\tau_{6}\right.$ from Table 2$)$ for $\operatorname{TTF-BTD}(\mathrm{R})_{2}(\mathrm{R}=\mathrm{H}, \mathrm{Br}, \mathrm{CN})$ in Toluene and $\mathrm{DMF}^{a}$

\begin{tabular}{lcc}
\multicolumn{1}{c}{ sample } & Em. peak $\left(\lambda_{\mathrm{Max}}^{\mathrm{Em}}\right)(\mathrm{nm})$ & BCT time $\left(\tau_{6}\right)(\mathrm{ps})$ \\
$\mathrm{H}$ in toluene & $675^{\mathrm{a}}$ & $410 \pm 50$ \\
$\mathrm{H}$ in DMF & $>690^{\mathrm{b}}$ & $570 \pm 200$ \\
$\mathrm{Br}$ in toluene & $680^{\mathrm{c}}$ & $870 \pm 10$ \\
$\mathrm{Br}$ in DMF & $750^{\mathrm{c}}$ & $124 \pm 1$ \\
$\mathrm{CN}$ in toluene & $>750^{\mathrm{d}}\left(890^{\mathrm{e}}\right)$ & $35.1 \pm 0.2$ \\
$\mathrm{CN}$ in DMF & $>800^{\mathrm{d}}\left(1100^{\mathrm{e}}\right)$ & $6.12 \pm 0.07$
\end{tabular}

${ }^{a}$ Values of $\lambda_{\text {Max }}^{\mathrm{Em}}$ are estimated from previous figures ( ${ }^{\text {a }}$ Figures 4 and S6, ${ }^{\mathrm{b}}$ Figures 5 and S6, and ${ }^{\mathrm{d}}$ Figures 8 and S8) in the literature $\left({ }^{\mathrm{c}}\right.$ ref 32) or $^{\mathrm{e}}$ calculated according to eq 3 (see eq S11 and the relative discussion in the SI).

and stabilization of the phototransferred electron due to slow relaxation of the molecular configuration.

Concerning the origin of the ESA bands, the overall spectral structure is almost the same throughout the series $\mathrm{H}-\mathrm{Br}-\mathrm{CN}$, suggesting that both the higher and lower ESA bands are very likely intraligand (IL) transitions of the oxidized $\mathrm{TTF}^{\circ+-}$ moiety, rather than the $\mathrm{BTD}^{\bullet-}$ one. More insight can be gained correlating the spectral features of TA spectra with those of DEC spectra in Figures 2 and S1, S2. Even though the solvents are different, this comparison corroborates the dominant role of IL transitions of the $\mathrm{TTF}^{\bullet+}$ group in the ESA signal. Both experimental data sets show indeed the following: a positive band peaked at $440 \mathrm{~nm}(500 \mathrm{~nm}$ for $\mathrm{CN})$, a negative bleaching at $490 / 520 / 660 \mathrm{~nm}(\mathrm{H} / \mathrm{Br} / \mathrm{CN}$, respectively), and a broadband ESA at $\lambda>550 \mathrm{~nm}$ for $\mathrm{H}$ and $\mathrm{Br}$. Conversely, the comparison with the DEC spectrum of $\mathrm{BTD}^{\bullet-}$ suggests that only the small shoulder at $390 \mathrm{~nm}$ in TA spectra could be related to IL signals from $\mathrm{BTD}^{\bullet-}$ group. In this respect, the $M_{0}$ analysis would rather corroborate that earlier dynamics in this spectral range are dominated by a hot $\mathrm{TTF}^{\bullet+}$ moiety. Finally, an IL character can also explain a lesser effect of solvent polarity on the spectral position of the ESA band. On the basis of these three arguments, we can assign TA ESA bands mainly to intraligand (IL) transitions localized on the $\mathrm{TTF}^{\bullet+}$ group with a small contribution from the $\mathrm{BTD}^{\bullet+}$ one (Figure S12).

The other remarkable result is the dominant role of solvent polarity on the CS lifetime: a polar environment causes a dramatic shortening of the CS state by almost 1 order of magnitude in $\mathrm{CN}$ (from 35 to $6 \mathrm{ps)}$ ) and $\mathrm{Br}$ ( 870 to $124 \mathrm{ps}$ ). The $\mathrm{H}$ complex is less affected but follows this trend (see Figure 6 or Table S1, for instance). Also notable is an SE Stokes shift of $0.7 \mathrm{eV}$ due to polar solvation only, in agreement with the big photoinduced permanent dipole $(20 \mathrm{D}){ }^{36}$

But the most intriguing outcome is that, contrary to expectations, the lifetime of the CS state decreases in more polar environments and with more electronegative acceptors (Figure 3). Indeed, we would have anticipated that a more electron-withdrawing acceptor and a more polar solvent stabilize the charge on the acceptor, resulting in a longer CS lifetime. Conversely, it shows a strong correlation with the energy gap between the equilibrated excited state and the ground one (see Table 3 and Figure S13).

To rationalize how solvent polarity can modulate an intramolecular process in such a strong and counterintuitive way, we propose that solvation changes the relative energy ordering of the excited states to make the photoexcited CS state more resonant with intermediate states with a non-CS character, which allows nonradiative BCT and relaxations to the GS. Indeed, these intermediate states should have a smaller permanent electric dipole because of the non-CS character and then they should undergo much less energy relaxation than the photoexcited ICT state, the latter being indirectly confirmed by the aforesaid pronounced SE shift. On the basis of these considerations, we proposed the scheme in Figure 9 (a scheme including also higher states responsible for the TA activity is depicted in Figure S12).

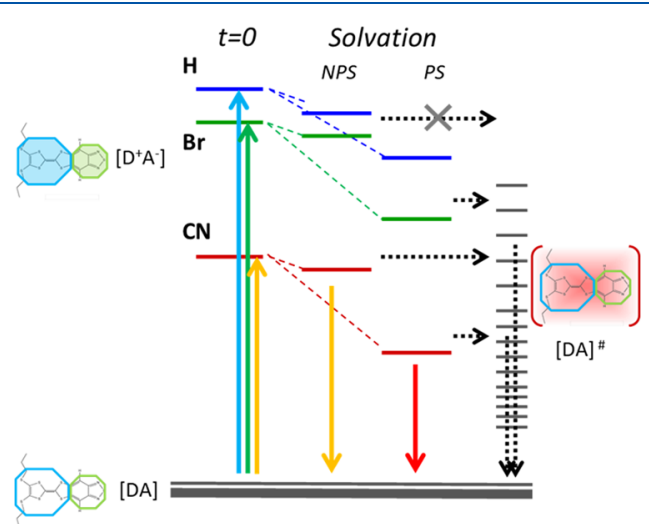

Figure 9. Simplified energy level diagram and photocycle for all systems upon excitation into the lowest ICT excited state, showing explicitly the effect of solvent relaxation (see for comparison Figures S11 and S12). Nomenclature: PS and NPS refer to polar and nonpolar solvents, respectively; D and A stand for donor and acceptor, namely, TTF and BTD moieties, in that order; superscripted \# identifies hot states; solid and dashed lines represent radiative and nonradiative transitions, respectively; blue and green shadings specify one-electron oxidation and reduction of the highlighted group; red shading refers to vibrationally excited (hot) states.

\section{CONCLUSIONS}

In this article, we report on the photoinduced charge separation dynamics in a recently synthesized family of metal-free tunable donor-acceptor systems, ${ }^{32,36}$ where two redox-active molecules are fused into a compact and planar structure upon annulation of a tetrathiafulvalene to a benzothiadiazole as a donor and acceptor, respectively. The former is a strong electron donor at relatively low oxidation potential values, whereas the latter is a well-known electrondeficient building block for semiconductor polymers.

We found an outstanding tunability of the lifetime of the photoinduced charge separation by more than 2 orders of magnitude (from 6 to $900 \mathrm{ps}$ ) upon minimal changes of the periphery residues of the acceptor and polarity of the environment. This is another manifestation of the peculiar behavior of this family of donor-acceptor dyads, which indeed shows an extended tunability in many physical properties, when compared with other $\pi$-conjugated systems and coordination complexes.

This study proves that the outstanding tunability of steadystate photophysics (absorption and emission transition frequencies, as reported in Tables S3 and 3, but also, for instance, redox properties ${ }^{30,32}$ ) goes hand in hand with an exceptional tunability of the lifetime of charge separation states, giving a new class of electronically versatile materials 
whose physicochemical properties can be tuned via a targeted substitution or suitable choice of local electrostatics.

Remarkably, the dependence of the CS lifetime on both the solvent polarity and on the acceptor electronegativity contradicts any argument based on the electrostatic stabilization of the charge on the acceptor. To rationalize how solvent polarity and rather small changes in the acceptor electron affinity can modulate an intramolecular process in such a pronounced way, we propose that solvation and acceptor electronegativity shift the energies of excited states with a CS character (Figure 1 and Table 3) whereas non-CS states are only weakly influenced. Accordingly, the photoexcited CS state is more or less resonant with intermediate states with a non-CS character (very likely hot states of the ground $\operatorname{state}^{36}$ ), which allow nonradiative relaxations to the ground state. This mechanism is corroborated by the correlation between the $\mathrm{BCT}$ rates and the emission frequency (Table 3 and Figure S13).

A further remarkable outcome of this study follows from a detailed investigation of the formation and stabilization of the CS state: we observe that even though the photoexcited transition leads directly to a charge-transfer state, only a fraction of charge is instantaneously localized on the acceptor, whereas the remaining fraction is transferred in the course of sub-picosecond intramolecular relaxation processes and solvent dielectric reorganization. Since an analogous result was recently reported for metal complexes, ${ }^{14,52,53}$ its observation also in small metal-free D-A dyads could suggest this to be a more universal phenomenon when charge-transfer states are photoexcited. However, to draw more conclusive results, more experimental and computational evidence is necessary.

Such a tunability in many physical properties motivates the application of fused $\mathrm{D}-\mathrm{A}$ systems in the field of molecular electronics or nonlinear optics, ${ }^{18,28,33-35}$ whereas a so enhanced sensitivity to local electrostatics could pave the way to applications as switchable photosensitizers, light emissive sensors for molecular electrostatics, etc. In a perspective view on the frontiers of molecular electronics and photocatalysis, it is evident that to achieve high catalytic performances, it is necessary to control local electrostatics and timing with respect to other processes. This class of dyads provides exceptional versatility to the generated local fields or available charges with a well-defined timing and to fine tune energy gaps with respect to other functional blocks.

\section{ASSOCIATED CONTENT}

\section{S Supporting Information}

The Supporting Information is available free of charge on the ACS Publications website at DOI: 10.1021/acs.jpcc.8b11066.

Derivation of eq 3, spectro-electrochemical measurements on TTF-BTD $(\mathrm{Br})_{2}$ and TTF-BTD $(\mathrm{CN})_{2}$, transient absorption setup and data acquisition, supplementary transient absorption spectra and kinetics, singular value decomposition and global fit (SVD-GF) analysis, charge-transfer quantum yield, spectral moment analysis, energetics, and photocycle (PDF)

\section{AUTHOR INFORMATION}

\section{Corresponding Author}

*E-mail: andrea.cannizzo@iap.unibe.ch.

\section{ORCID}

Egmont Rohwer: 0000-0002-9123-2338

Andrea Cannizzo: 0000-0002-2325-0112

\section{Present Addresses}

"Department of Chemistry, University of Fribourg, Chemin du Musée 9, CH-1700 Fribourg, Switzerland (J.H.).

${ }^{\perp}$ College of Chemistry, Chemical Engineering and Material Science, Shandong Normal University, Jinan 250014, P. R. China (Y.G.).

\section{Author Contributions}

The manuscript was written through contributions of all authors. All authors have given approval to the final version of the manuscript.

\section{Notes}

The authors declare no competing financial interest.

\section{ACKNOWLEDGMENTS}

This research was supported by the NCCR MUST research instrument of the Swiss National Science Foundation and the European Commission (EC) FP7 ITN "MOLESCO” (project no. 606728).

\section{REFERENCES}

(1) Vougioukalakis, G. C.; Philippopoulos, A. I.; Stergiopoulos, T.; Falaras, P. Contributions to the Development of Ruthenium-Based Sensitizers for Dye-Sensitized Solar Cells. Coord. Chem. Rev. 2011, $255,2602-2621$.

(2) Kinoshita, T.; Dy, J. T.; Uchida, S.; Kubo, T.; Segawa, H. Wideband Dye-Sensitized Solar Cells Employing a PhosphineCoordinated Ruthenium Sensitizer. Nat. Photonics 2013, 7, 535-539.

(3) Yella, A.; Lee, H.-W.; Tsao, H. N.; Yi, C.; Chandiran, A. K.; Nazeeruddin, M. K.; Diau, E. W.-G.; Yeh, C.-Y.; Zakeeruddin, S. M.; Graetzel, M. Porphyrin-Sensitized Solar Cells with Cobalt (Ii/Iii)Based Redox Electrolyte Exceed 12\% Efficiency. Science 2011, 334, 629-634.

(4) Imahori, H.; Umeyama, T.; Ito, S. Large П-Aromatic Molecules as Potential Sensitizers for Highly Efficient Dye-Sensitized Solar Cells. Acc. Chem. Res. 2009, 42, 1809-1818.

(5) Elvington, M.; Brown, J.; Arachchige, S. M.; Brewer, K. J. Photocatalytic Hydrogen Production from Water Employing a $\mathrm{Ru}$, $\mathrm{Rh}, \mathrm{Ru}$ Molecular Device for Photoinitiated Electron Collection. J. Am. Chem. Soc. 2007, 129, 10644-10645.

(6) Fihri, A.; Artero, V.; Pereira, A.; Fontecave, M. Efficient H-2Producing Photocatalytic Systems Based on Cyclometalated Iridiumand Tricarbonylrhenium-Diimine Photosensitizers and Cobaloxime Catalysts. Dalton Trans. 2008, 5567-5569.

(7) Fihri, A.; Artero, V.; Razavet, M.; Baffert, C.; Leibl, W.; Fontecave, M. Cobaloxime-Based Photocatalytic Devices for Hydrogen Production. Angew. Chem., Int. Ed. 2008, 47, 564-567.

(8) Inagaki, A.; Akita, M. Visible-Light Promoted Bimetallic Catalysis. Coord. Chem. Rev. 2010, 254, 1220-1239.

(9) Lei, P.; Hedlund, M.; Lomoth, R.; Rensmo, H.; Johansson, O.; Hammarstrom, L. The Role of Colloid Formation in the Photoinduced H-2 Production with a Ru-Ii-Pd-Ii Supramolecular Complex: A Study by Gc, Xps, and Tem. J. Am. Chem. Soc. 2008, 130, 26-27.

(10) Losse, S.; Vos, J. G.; Rau, S. Catalytic Hydrogen Production at Cobalt Centres. Coord. Chem. Rev. 2010, 254, 2492-2504.

(11) Ozawa, H.; Sakai, K. Photo-Hydrogen-Evolving Molecular Devices Driving Visible-Light-Induced Water Reduction into Molecular Hydrogen: Structure-Activity Relationship and Reaction Mechanism. Chem. Commun. 2011, 47, 2227-2242.

(12) Probst, B.; Rodenberg, A.; Guttentag, M.; Hamm, P.; Alberto, R. A Highly Stable Rhenium-Cobalt System for Photocatalytic H-2 Production: Unraveling the Performance-Limiting Steps. Inorg. Chem. 2010, 49, 6453-6460.

(13) Tschierlei, S.; Karnahl, M.; Presselt, M.; Dietzek, B.; Guthmuller, J.; Gonzalez, L.; Schmitt, M.; Rau, S.; Popp, J. Photochemical Fate: The First Step Determines Efficiency of H-2 
Formation with a Supramolecular Photocatalyst. Angew. Chem., Int. Ed. 2010, 49, 3981-3984.

(14) Cannizzo, A.; Blanco-Rodriguez, A. M.; El Nahhas, A.; Sebera, J.; Zalis, S.; Vlcek, A.; Chergui, M. Femtosecond Fluorescence and Intersystem Crossing in Rhenium(I) Carbonyl-Bipyridine Complexes. J. Am. Chem. Soc. 2008, 130, 8967-8974.

(15) Cannizzo, A.; Milne, C. J.; Consani, C.; Gawelda, W.; Bressler, C.; van Mourik, F.; Chergui, M. Light-Induced Spin Crossover in $\mathrm{Fe}(\mathrm{Ii})$-Based Complexes: The Full Photocycle Unraveled by Ultrafast Optical and X-Ray Spectroscopies. Coord. Chem. Rev. 2010, 254, $2677-2686$.

(16) Cannizzo, A.; van Mourik, F.; Gawelda, W.; Zgrablic, G.; Bressler, C.; Chergui, M. Broadband Femtosecond Fluorescence Spectroscopy of $[\mathrm{Ru}(\mathrm{Bpy})(3)](2+)$. Angew. Chem., Int. Ed. 2006, 45, 3174-3176.

(17) Lee, C. P.; Li, C. T.; Ho, K. C. Use of Organic Materials in Dye-Sensitized Solar Cells. Mater. Today 2017, 20, 267-283.

(18) Wu, Y.; Zhu, W. Organic Sensitizers from D-П-a to D-a-П-A: Effect of the Internal Electron-Withdrawing Units on Molecular Absorption, Energy Levels and Photovoltaic Performances. Chem. Soc. Rev. 2013, 42, 2039-2058.

(19) Gratzel, M. Recent Advances in Sensitized Mesoscopic Solar Cells. Acc. Chem. Res. 2009, 42, 1788-1798.

(20) Kakiage, K.; Aoyama, Y.; Yano, T.; Oya, K.; Fujisawa, J.; Hanaya, M. Highly-Efficient Dye-Sensitized Solar Cells with Collaborative Sensitization by Silyl-Anchor and Carboxy-Anchor Dyes. Chem. Commun. 2015, 51, 15894-15897.

(21) Mishra, A.; Fischer, M. K. R.; Bauerle, P. Metal-Free Organic Dyes for Dye-Sensitized Solar Cells: From Structure: Property Relationships to Design Rules. Angew. Chem., Int. Ed. 2009, 48, 2474-2499.

(22) Koumura, N.; Wang, Z.-S.; Mori, S.; Miyashita, M.; Suzuki, E.; Hara, K. Alkyl-Functionalized Organic Dyes for Efficient Molecular Photovoltaics. J. Am. Chem. Soc. 2006, 128, 14256-14257.

(23) O’Regan, B. C.; Durrant, J. R. Kinetic and Energetic Paradigms for Dye-Sensitized Solar Cells: Moving from the Ideal to the Real. Acc. Chem. Res. 2009, 42, 1799-1808.

(24) Amacher, A.; Yi, C.; Yang, J.; Bircher, M. P.; Fu, Y.; Cascella, M.; Gratzel, M.; Decurtins, S.; Liu, S.-X. A Quinoxaline-Fused Tetrathiafulvalene-Based Sensitizer for Efficient Dye-Sensitized Solar Cells. Chem. Commun. 2014, 50, 6540-6542.

(25) Bergkamp, J. J.; Decurtins, S.; Liu, S. X. Current Advances in Fused Tetrathiafulvalene Donor-Acceptor Systems. Chem. Soc. Rev. 2015, 44, 863-874.

(26) Canevet, D.; Salle, M.; Zhang, G.; Zhang, D.; Zhu, D. Tetrathiafulvalene (Ttf) Derivatives: Key Building-Blocks for Switchable Processes. Chem. Commun. 2009, 2245-2269.

(27) Liu, S. X.; Dolder, S.; Rusanov, E. B.; Stoeckli-Evans, H.; Decurtins, S. Novel Unsymmetrically Functionalized Bedt-Ttf Derivatives: Synthesis, Crystal Structure and Electrochemical Characterization. C. R. Chim. 2003, 6, 657-662.

(28) Justin Thomas, K. R.; Lin, J. T.; Velusamy, M.; Tao, Y. T.; Chuen, C. H. Color Tuning in Benzo[1,2,5]Thiadiazole-Based Small Molecules by Amino Conjugation/Deconjugation: Bright Red-LightEmitting Diodes. Adv. Funct. Mater. 2004, 14, 83-90.

(29) Wang, X.; Sun, Y.; Chen, S.; Guo, X.; Zhang, M.; Li, X.; Li, Y.; Wang, H. Effects of $\Pi$-Conjugated Bridges on Photovoltaic Properties of Donor-П-Acceptor Conjugated Copolymers. Macromolecules 2012, $45,1208-1216$.

(30) Amacher, A.; Luo, H.; Liu, Z.; Bircher, M.; Cascella, M.; Hauser, J.; Decurtins, S.; Zhang, D.; Liu, S.-X. Electronic Tuning Effects Via Cyano Substitution of a Fused TetrathiafulvaleneBenzothiadiazole Dyad for Ambipolar Transport Properties. RSC Adv. 2014, 4, 2873-2878.

(31) Geng, Y.; Pfattner, R.; Campos, A.; Hauser, J.; Laukhin, V.; Puigdollers, J.; Veciana, J.; Mas-Torrent, M.; Rovira, C.; Decurtins, S.; et al. A Compact Tetrathiafulvalene-Benzothiadiazole Dyad and Its Highly Symmetrical Charge-Transfer Salt: Ordered Donor П-Stacks
Closely Bound to Their Acceptors. Chem. - Eur. J. 2014, 20, 71367143.

(32) Pop, F.; Amacher, A.; Avarvari, N.; Ding, J.; Daku, L. M. L.; Hauser, A.; Koch, M.; Hauser, J.; Liu, S.-X.; Decurtins, S. Tetrathiafulvalene-Benzothiadiazoles as Redox-Tunable Donor-Acceptor Systems: Synthesis and Photophysical Study. Chem. - Eur. J. 2013, 19, 2504-2514.

(33) Bendikov, M.; Wudl, F.; Perepichka, D. F. Tetrathiafulvalenes, Oligoacenenes, and Their Buckminsterfullerene Derivatives: The Brick and Mortar of Organic Electronics. Chem. Rev. 2004, 104, 4891-4945.

(34) Martín, N. Tetrathiafulvalene: The Advent of Organic Metals. Chem. Commun. 2013, 49, 7025-7027.

(35) Geng, Y.; Pfattner, R.; Campos, A.; Wang, W.; Jeannin, O.; Hauser, J.; Puigdollers, J.; Bromley, S. T.; Decurtins, S.; Veciana, J.; et al. Homo Stabilisation in Pi-Extended Dibenzotetrathiafulvalene Derivatives for Their Application in Organic Field-Effect Transistors. Chem. - Eur. J. 2014, 20, 16672-16679.

(36) Rohwer, E. J.; Akbarimoosavi, M.; Meckel, S. E.; Liu, X.; Geng, Y.; Lawson Daku, L. M.; Hauser, A.; Cannizzo, A.; Decurtins, S.; Stanley, R. J.; et al. Dipole Moment and Polarizability of Tunable Intramolecular Charge Transfer States in Heterocyclic П-Conjugated Molecular Dyads Determined by Computational and Stark Spectroscopic Study. J. Phys. Chem. C 2018, 122, 9346-9355.

(37) Ly, H. K.; Wisitruangsakul, N.; Sezer, M.; Feng, J. J.; Kranich, A.; Weidinger, I. M.; Zebger, I.; Murgida, D. H.; Hildebrandt, P. Electric-Field Effects on the Interfacial Electron Transfer and Protein Dynamics of Cytochrome C. J. Electroanal. Chem. 2011, 660, 367376.

(38) Boxer, S. G. Stark Realities. J. Phys. Chem. B 2009, 113, 29722983.

(39) Ohta, N.; Koizumi, M.; Umeuchi, S.; Nishimura, Y.; Yamazaki, I. External Electric Field Effects on Fluorescence in an Electron Donor and Acceptor System: Ethylcarbazole and Dimethyl Terephthalate in Pmma Polymer Films. J. Phys. Chem. 1996, 100, 1646616471.

(40) Lao, K. Q.; Franzen, S.; Steffen, M.; Lambright, D.; Stanley, R.; Boxer, S. G. Effects of Applied Electric-Fields on the Quantum Yields for the Initial Electron-Transfer Steps in Bacterial Photosynthesis.2. Dynamic Stark-Effect. Chem. Phys. 1995, 197, 259-275.

(41) Lockhart, D. J.; Kirmaier, C.; Holten, D.; Boxer, S. G. ElectricField Effects on the Initial Electron-Transfer Kinetics in Bacterial Photosynthetic Reaction Centers. J. Phys. Chem. 1990, 94, 69876995.

(42) Wahadoszamen, M.; Nakabayashi, T.; Kang, S.; Imahori, H.; Ohta, N. External Electric Field Effects on Absorption and Fluorescence Spectra of a Fullerene Derivative and Its Mixture with Zinc-Tetraphenylporphyrin Doped in a Pmma Film. J. Phys. Chem. B 2006, 110, 20354-20361.

(43) Schindler, F.; Lupton, J. M.; Muller, J.; Feldmann, J.; Scherf, U. How Single Conjugated Polymer Molecules Respond to Electric Fields. Nat. Mater. 2006, 5, 141-146.

(44) Bublitz, G. U.; Boxer, S. G. Stark Spectroscopy: Applications in Chemistry, Biology, and Materials Science. Annu. Rev. Phys. Chem. 1997, 48, 213-242.

(45) Franzen, S.; Stanley, R. J. A Theoretical Explanation for Quantum Yield Failure in Bacterial Photosynthetic Reaction Centers. Chem. Phys. 2002, 276, 115-127.

(46) Pudlak, M.; Pincak, R. Influence of the Electric Field on the Electron Transport in Photosynthetic Reaction Centers. Eur. Phys. J. E: Soft Matter Biol. Phys. 2011, 34, No. 22.

(47) Lewis, N. S.; Nocera, D. G. Powering the Planet: Chemical Challenges in Solar Energy Utilization. Proc. Natl. Acad. Sci. U.S.A. 2006, 103, 15729-15735.

(48) Balzani, V.; Credi, A.; Venturi, M. Processing Energy and Signals by Molecular and Supramolecular Systems. Chem. - Eur. J. 2008, 14, 26-39. 
(49) Horng, M. L.; Gardecki, J. A.; Papazyan, A.; Maroncelli, M. Subpicosecond Measurements of Polar Solvation Dynamics Coumarin-153 Revisited. J. Phys. Chem. 1995, 99, 17311-17337.

(50) Lakowicz, J. R. Principles of Fluorescence Spectroscopy, 2nd ed.; Kluwer Academic/Plenum: New York, 1999; Vol. xxiii, p 698.

(51) Zálǐ̌, S.; Consani, C.; El Nahhas, A.; Cannizzo, A.; Chergui, M.; Hartl, F.; Vlcek, A. Origin of Electronic Absorption Spectra of Mlct-Excited and One-Electron Reduced 2,2'-Bipyridine and 1,10Phenanthroline Complexes. Inorg. Chim. Acta 2011, 374, 578-585.

(52) Frei, F.; Rondi, A.; Espa, D.; Mercuri, M. L.; Pilia, L.; Serpe, A.; Odeh, A.; Van Mourik, F.; Chergui, M.; Feurer, T.; et al. Ultrafast Electronic and Vibrational Relaxations in Mixed-Ligand DithioneDithiolato Ni, Pd, and Pt Complexes. Dalton Trans. 2014, 43, 1766617676.

(53) Rondi, A.; Rodriguez, Y.; Feurer, T.; Cannizzo, A. SolvationDriven Charge Transfer and Localization in Metal Complexes. Acc. Chem. Res. 2015, 48, 1432-1440. 